\title{
COVID-19 infection and diabetes mellitus
}

\begin{abstract}
Diabetes mellitus (among other conditions) has received attention as an important comorbidity predisposing to a COVID-19 infection and its control determining prognosis from this infection. We have evaluated important associations about how diabetes mellitus (and hyperglycaemia) can affect the course of a COVID-19 infection as well as reviewing the impact of the COVID-19 pandemic on diabetic services. Existing literatures have captured aspects of the interaction of these two entities. We hope to give a vantage view for ease of knowledge.
\end{abstract}

Keywords: COVID-19, diabetes mellitus
Volume 7 Issue 4 - 2020

\author{
Alamin Alkundi, Rabiu Momoh \\ William Harvey Hospital Ashford, United Kingdom
}

Correspondence: Dr. Alamin Alkundi , Consultant Endocrinologist, William Harvey Hospital, Ashford, Kent, United Kingdom, Email alain.alkundi@nhs.net

Received: November 13, 2020 | Published: November 25, 2020
One of the most frequently associated clinical entities in recent times is the risk and prognostic correlation drawn between diabetes mellitus and COVID-19 infection. Large volumes of published literature have made this association as well as trying to explore the underlying pathophysiology responsible for the association. ${ }^{1}$ We hope to give a vantage view on the association between these two clinical entities. The COVID-19 infection that started as an epidemic in the Wuhan area of China has achieved a pandemic status, with documented evidence of infection from the paediatric to the geriatric age groups. ${ }^{2}$ Background medical conditions have been identified as predisposing factors to contracting this disease, of which diabetes mellitus rates among the early counters. It may also be seen in combination with other prominent co-morbidities like hypertension, chronic obstructive pulmonary disease, morbid obesity, heart failure, renal failure, liver failure, organ transplants, advanced cancers, chemotherapy and radiotherapy administration, HIV/AIDS infection, among others. ${ }^{3}$ The COVID-19 virus (an envelope, positive singlestranded RNA viruses) is largely transmitted via inhalation with time to symptom onset of up to 14 days commonly. ${ }^{4}$ Common symptoms associated with the COVID-19 include fever, cough, shortness of breath, fatigue, myalgia, loss of sense of smell or taste. Other rare extra-pulmonary but described features of COVID-19 infection include headache, diarrhea, seizure, conjunctivitis, nephritis, or acute kidney injury. ${ }^{5}$ Diabetes mellitus is a clinical condition of impaired glucose, protein, and fat metabolism due to either deficient insulin production or resistance to insulin in the body. ${ }^{6} \mathrm{~A}$ higher prevalence of diabetes mellitus in developed countries is known but recently more noticeable higher incidence rates in low and middle-income countries ( where death rate from diabetes is higher). Type 2 diabetes mellitus accounts (a condition of impaired insulin sensitivity) accounts for about $90 \%$ of diagnosed diabetes mellitus worldwide. ${ }^{7}$ Jing Y et al., ${ }^{8}$ while conducting a meta-analytical study on literature works done on COVID-19 and diabetics, made a deduction of a prevalence rate of roughly $10 \%$ background diabetes among COVID-19 infection count. ${ }^{8}$ The major pathophysiology of the SARS-COV-2 virus in human hosts is the severe pro-inflammatory response stimulated by this virus upon entry to the human body, described in a more scientific term as the "cytokine storm". 9 The impaired innate and humoral immune systems in diabetics with poor glycaemic control have also been alluded to as one of the possible causes of higher predisposition and severity of COVID-19 infection in diabetics.

The cytokine storm earlier described has been found to be exaggerated in diabetics with COVID-19 infection. ${ }^{10}$ As of 2014 (based on data by the World Health Organisation), there were about 422 million individuals living with diabetes. ${ }^{11}$ It remains to be figured out what the figure for the worldwide prevalence of diabetes mellitus is in 2020 in relation to the ongoing pandemic. As of the 19th August 2020, there have been more than 22 million confirmed cases of COVID-19 infection worldwide, and about 770,000 deaths recorded. ${ }^{12}$ About 3.8 million individuals live with diabetes in the United Kingdom, as noted by figures released by the UK Government, and up to $90 \%$ of these patients have Type 2 diabetes mellitus. ${ }^{13}$ Alkundi et al., ${ }^{14}$ while describing the clinical profile and outcome of COVID-19 infection among diabetics at a district general hospital in the United Kingdom, made notable conclusions. As described above, they found that diabetics are at risk of a longer hospital stay (14.4 ( $\mathrm{SD} \pm 9.6)$ days) with COVID-19 infection than non-diabetics (9.8 ( $\mathrm{SD} \pm 17.1)$ days, $\mathrm{p}<$ $0.0001)$. They also found that there were more male diabetics $(43.4 \%)$ admitted with COVID-19 infections than female diabetics (27.6\%) $\mathrm{p}$ value $=0.016$. They found more Type $2 \mathrm{DM}$ patients compared to Type 1 with COVID-19 infection. They also found that older patients with diabetes were at a higher risk of mortality from COVID-19 than younger age groups. ${ }^{14}$ Ashish Kumar et al., ${ }^{15}$ found out that diabetics were twice as likely to have mortality as an outcome of COVID-19 infection than non-diabetics. ${ }^{15}$

While analysing nationwide England data, Naomi Holman et al., ${ }^{16}$ in their article (focused on addressing the association between Types 1 and 2 diabetes mellitus and COVID-19 mortality), also concluded that both diabetes types accounted for increased odds for in-hospital deaths from COVID-19 related hospital admissions. They also highlighted the heightened COVID-19 mortality risk associated with longstanding glycaemic control, as evident in glycaemic hemoglobin values of diabetic patients with COVID-19 infection. ${ }^{16}$ Patients have been described to have presented with diabetic ketoacidosis symptoms as reasons for hospital admission for onward COVID-19 diagnosis. Deranged blood sugar control has been associated with COVID-19 infection. Where diabetic ketoacidosis complicates severe COVID-19 infection, consideration should be given to care in an intensive care unit with multidisciplinary care involving the endocrinologist. Juyi Li et., ${ }^{17}$ all acceded to the consideration that COVID-19 could induce ketosis and ketoacidosis, as well as causing diabetic keto-acidosis among diabetics with COVID-19 infection. ${ }^{17}$ Mark Aloysius et al., ${ }^{18}$ raised consideration about the potential of the SARS-COV-2 virus to cause acute pancreatitis, alluding to the possible presence of the ACE2 receptor for COVID-19 intracellular entry expressed on the pancreas. This was corroborated in their case report in an individual 
with severe respiratory COVID-19 manifestation and features of acute pancreatitis. ${ }^{18}$ The involvement of this virus with the beta- islet cells of the pancreas remains to be fully described. The interesting interplay between a severe COVID-19 infection and an onward development of new diabetes mellitus has started to be alluded to. ${ }^{19}$ Hyperglycaemia by itself, independent of a diagnosis of diabetes mellitus, has also been associated with a severe COVID-19 manifestation. ${ }^{20}$ Akthar Hussain et al., ${ }^{19}$ in his publication, reported the possibility of hypoglycemia complicating hydroxychloroquine treatment of COVID-19 infections in diabetics. ${ }^{19}$

Preventing the COVID-19 virus transmission to diabetics in the first place should be the goal. Shielding measures were recommended for diabetics at the start of lockdown measures in the United Kingdom, and diabetics were considered as moderate-risk individuals to contracting COVID-19. ${ }^{21}$ Other preventive measures such as personal hygiene (handwashing, avoiding touching face with hands, personal protective equipment, and social distancing) are some ways to keep the COVID-19 infection at bay for diabetics. Ensuring blood sugar control could serve as a primary preventive strategy against COVID-19 infection among diabetics. ${ }^{22}$ The deranged blood sugar experienced during a severe COVID-19 infection may be best managed with the use of insulin and Dipeptidyl peptidase- 4 inhibitors. In the setting of dehydration and acute kidney injury complication COVID-19 infection in a type 2 diabetic, blood glucose control can be addressed by removing a lactic acidosis former e.g. metformin from list of medications and using insulin. ${ }^{22}$ Treatment options that would dampen the effect of the cytokine storm in patients could prevent the vital organ dysfunctions that COVID-19 is known for as well as keeping glucose control within checks. ${ }^{22}$ Significant interruptions in diabetic services have been recorded in hospitals due to lockdown measures for COVID-19 control. It remains to be fully described what the effect of extended lockdown measures did have on services like foot clinics, diabetic retinopathy services and other routine clinic services for glucose control in diabetics in different regions of the world. ${ }^{23}$ Measures like telephone and video consultations replaced physical clinic attendance during the hit of the COVID-19 spread in many world areas. Fail-proof treatment protocol for the management of severe COVID-19 infection has eluded the world till the time of this publication. Preventing COVID-19 infection among diabetics and ensuring their glycaemic control in the face of the ongoing pandemic will help prevent undesired outcomes.

\section{Acknowledgments}

None.

\section{Conflicts of interest}

The authors declare that there are no conflicts of interest.

\section{Funding}

None.

\section{References}

1. Chrysi K, Anastasios T, Ioanna E, et al. Clinical management of diabetes mellitus in the era of COVID-19: practical issues, peculiarities and concerns. J Clin Med. 2020;9(7):2288.
2. Saima H, Mohammad Y, Gulab K, et al. Novel coronavirus disease (COVID-19): a pandemic (epidemiology, pathogenesis and potential therapeutics). New Microbes New Infect. 2020;35:100679.

3. Andrew C, Mark J, Charlotte W, et al. Global, regional, and national estimates of the population at increased risk of severe COVID-19 due to underlying health conditions in 2020: a modelling study. Lancet Glob Health. 2020;8(8):e1003-e1017.

4. Yinon MB, Avi F, Rob P, et al. SARS-CoV-2 (COVID-19) by the numbers. Elife. 2020;9:e57309.

5. Aakriti G, Mahesh VM, Kartik S, et al. Extrapulmonary manifestation of COVID-19. Nat Med. 2020;26(7):1017-1032.

6. ADA. Diagnosis and classification of diabetes mellitus. Diabetes Care. 2013;36 Suppl 1(Suppl 1):S67-S74.

7. Anjali DD, Marcie H, Mario S. Eidemiology of diabetes and diabetesrelated complications. Phys Ther. 2008;88(11):1254-1264.

8. Jing Y, Ya Z, Xi G, et al. Prevalence of comorbidities and its effects in patients infected with SARS-CoV-2: a systematic review and metaanalysis. Int J Infect Dis. 2020;94:91-95.

9. Lauren AH, Scott WC, Grant SS, et al. On the alert for cytokine storm: immunopathology in COVID-19. Arthritis Rheumatol. 2020;72(7):10591063.

10. Rimesh P, Sanjay KB. COVID-19 and diabetes mellitus: an unholy interaction of two epidemics. Diabetes Metab Syndr. 2020;14(4):513-517.

11. WHO. Diabetes.

12. WHO. WHO coronavirus disease (COVID-19) dashboard.

13. GOV.UK. 3.8 million people in England now have diabetes.

14. Alamin A, Ibrahim M, Abdelmajid M, et al. Clinical characteristics and outcomes of COVID-19 hospitalized patients with diabetes in the United Kingdom: a retrospective single centre study. Diabetes Res Clin Pract. 2020;165:108263.

15. Ashish K, Anil A, Praveen S, et al. Is diabetes mellitus associated with mortality and severity of COVID-19? a meta-analysis. Diabetes Metab Syndr. 2020;14(4):535-545.

16. Naomi H, Peter K, Partha K, et al. Risk factors for COVID-19-related mortality in people with type 1 and type 2 diabetes in England: a populationbased cohort study. Lancet Diabetes Endocrinol. 2020;8(10):823-833.

17. Juyi L, Xiufang W, Jian C, et al. COVID-19 infection may cause ketosis and ketoacidosis. Diabetes Obes Metab. 2020;10.1111/dom.14057.

18. Mark MA, Ashwin T, Anjalika G, et al. COVID-19 presenting as acute pancreatitis. Pancreatology. 2020;20(5):1026-1027.

19. Akhtar H, Bishwajit B, Nayla C. COVID-19 and diabetes: knowledge in progress. Diabetes Res Clin Pract. 2020;162:108142.

20. Brufsky A. Hyperglycemia, hydroxychloroquine, and the COVID-19 pandemic. J Med Virol. 2020;92(7):770-775.

21. NHS. Who's at higher risk from coronavirus.

22. Stefan RB, Francesco R, Kamlesh K, et al. Practical recommendations for the management of diabetes in patients with COVID-19. Lancet Diabetes Endocrinol. 2020;8(6):546-550.

23. Deborah JW, Fraser WG, Partha K, et al. Endocrinology in the time of covid-19: remodelling diabetes services and emerging innovation. Eur $J$ Endocrinol. 2020;183(2):G67-G77. 